\title{
d-wave Superconductivity in the Hubbard Model
}

\author{
Th. Maier ${ }^{1}$, M. Jarrell ${ }^{2}$, Th. Pruschke ${ }^{1}$, J. Keller $^{1}$ \\ ${ }^{1}$ Institut für Theoretische Physik, Universität Regensburg, 93040 Regensburg \\ ${ }^{2}$ Department of Physics, University of Cincinnati, Cincinnati, OH 45221-0011
}

(October 29, 2018)

The superconducting instabilities of the doped repulsive 2D Hubbard model are studied in the intermediate to strong coupling regime with help of the Dynamical Cluster Approximation (DCA). To solve the effective cluster problem we employ an extended Non Crossing Approximation (NCA), which allows for a transition to the broken symmetry state. At sufficiently low temperatures we find stable d-wave solutions with off-diagonal long range order. The maximal $T_{c} \approx 150 \mathrm{~K}$ occurs for a doping $\delta \approx 20 \%$ and the doping dependence of the transition temperatures agrees well with the generic high- $T_{c}$ phase diagram.

Introduction The discovery of high- $T_{c}$ superconductors has stimulated strong experimental and theoretical interest in the field of strongly correlated electron systems. After a decade of intensive studies we are still far from a complete understanding of the rich physics observed in high- $T_{c}$ cuprates [1]. Angle resolved photoemission experiments on doped materials show a $d$-wave anisotropy of the pseudogap in the superconducting state [2]. In underdoped materials even in the normal state this pseudogap persists [2,3], which is believed to cause the unusual non-Fermi-liquid behavior in the normal state. This emphasizes the importance of achieving a better understanding of the superconducting phase, i.e. the physical origin of the pairing mechanism, the nature of the pairing state and the character of low energy excitations.

On a phenomenological basis the $d$-wave normal state pseudogap as well as the transition to a superconducting state with a $d$-wave order parameter has been described within theories where short-ranged antiferromagnetic spin fluctuations mediate pairing in the cuprates 46. 6 .

On a microscopic level it is believed that the Hubbard model or closely related models like the t-J model should capture the essential physics of the high- $T_{c}$ cuprates [7]. However, despite years of intensive studies, these models remain unsolved except in one or infinite dimensions.

Finite size QMC calculations for the doped 2D Hubbard model in the intermediate coupling regime with Coulomb repulsion $U$ less than or equal to the bandwidth $W$, support the idea of a spin fluctuation driven interaction mediating $d$-wave superconductivity [4]. But the fermion sign problem limits these calculations to temperatures too high to observe a possible Kosterlitz-Thouless transition for the 2D system [4]. Another problem encountered in QMC calculations is their finite size character, which makes statements for the thermodynamic limit dependent on a scaling ansatz.

These limitations do not apply to approximate many particle methods like the Fluctuation Exchange Approximation (FLEX) [8,9]. Results of FLEX calculations for the Hubbard model are in agreement with QMC results, i.e. they show evidence for a superconducting state with $d$-wave order parameter at moderate doping for sufficiently low temperatures [8,9]. But the FLEX method as an approximation based on a perturbative expansion in $U$ breaks down in the strong coupling regime $U>W$, where $W$ is the bare bandwidth. On the other hand it is believed that a proper description of the high- $T_{c}$ cuprates in terms of the one-band Hubbard model requires $U>W$.

Calculations within the Dynamical Mean Field Approximation (DMFA) [10] can be performed in the strong coupling regime and take place in the thermodynamic limit. But the lack of non-local correlations inhibits a transition to a state with a non-local ( $d$-wave) order parameter. The recently developed Dynamical Cluster Approximation (DCA) 11 13 is a fully causal approach which systematically incorporates non-local corrections to the DMFA by mapping the lattice problem onto an embedded periodic cluster of size $N_{c}$. For $N_{c}=1$ the DCA is equivalent to the DMFA and by increasing the cluster size $N_{c}$ the dynamic correlation length can be gradually increased while the DCA solution remains in the thermodynamic limit.

Using a Nambu-Gorkov representation of the DCA we observe a transition to a superconducting phase in doped systems at sufficiently low temperatures. This occurs in the intermediate to strong coupling regime $U>W$ and the corresponding order parameter has $d$-wave symmetry.

Method A detailed discussion of the DCA formalism was given in previous publications [11 13 where it was shown to systematically restore momentum conservation at internal diagrammatic vertices which is relinquished by the DMFA. However, the DCA also has a simple physical interpretation based on the observation that the self energy is only weakly momentum dependent for systems where the dynamical intersite correlations have only short spatial range. The corresponding self-energy is a functional of the interaction $U$ and the Green function propagators. The latter may be calculated on a coarse grid of $N_{c}=L^{D}$ selected $\mathbf{K}$-points only, where $L$ is the 
linear dimension of the cluster of $\mathbf{K}$-points. According to Nyquist's sampling theorem [14], this sampling of the reciprocal space at intervals of $\Delta \mathbf{k}=2 \pi / L$ implies that the DCA incorporates nonlocal dynamical correlations with a spatial range $\lesssim L / 2$ and cuts off longer ranged dynamical correlations. Knowledge of the momentum dependence on a finer grid may be discarded to reduce the complexity of the problem. To this end the first Brillouin zone is divided into $N_{c}$ cells of size $(2 \pi / L)^{D}$ around the cluster momenta $\mathbf{K}$ (see Fig.11). The Green functions used to form the self-energy $\Sigma(\mathbf{K}, \omega)$ are coarse grained, or averaged over the momenta $\mathbf{K}+\tilde{\mathbf{k}}$ surrounding the

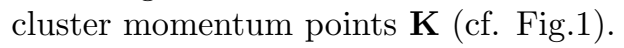

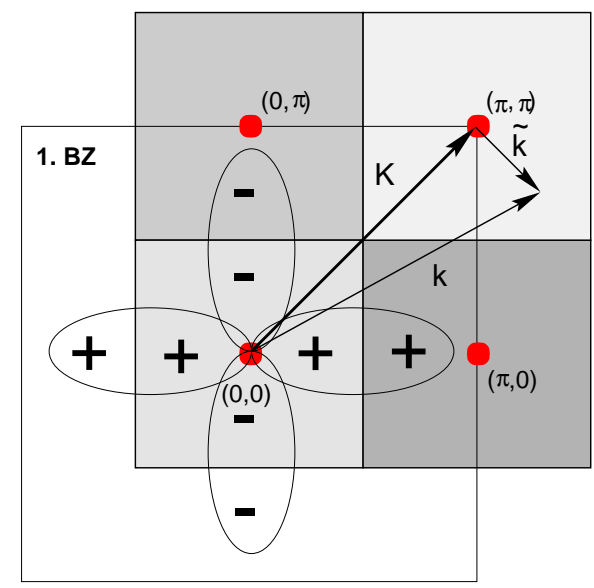

FIG. 1. Choice of the $N_{c}=4$ cluster $\mathbf{K}$-points (filled circles), corresponding coarse graining cells (shown by different fill patterns) and a sketch of the $d$-wave symmetry of the order parameter.

Thus, the coarse grained Green function is

$$
\hat{\bar{G}}(\mathbf{K}, \omega)=\frac{N_{c}}{N} \sum_{\tilde{\mathbf{k}}} \hat{G}(\mathbf{K}+\tilde{\mathbf{k}}, \omega),
$$

where the sum runs over all vectors $\mathbf{k}=\mathbf{K}+\tilde{\mathbf{k}}$ within a cell around the cluster momentum $\mathbf{K}$. Note that the choice of the coarse grained Green function has two well defined limits: For $N_{c}=1$ the sum over $\tilde{\mathbf{k}}$ runs over the entire Brillouin zone, $\hat{\bar{G}}$ is the local Green function, thus the DMFA algorithm is recovered. For $N_{c}=\infty$ the $\tilde{\mathbf{k}}$ summation vanishes and the DCA becomes equivalent to the exact solution. The dressed lattice Green function takes the form

$$
\hat{G}(\mathbf{k}, \omega)=\left(\omega \mathbb{1}-\epsilon_{\mathbf{k}} \tau_{3}-\hat{\Sigma}(\mathbf{K}, \omega)\right)^{-1},
$$

with the self-energy $\hat{\Sigma}(\mathbf{k}, \omega)$ approximated by the cluster self-energy $\hat{\Sigma}(\mathbf{K}, \omega)$. To allow for a possible transition to the superconducting state we utilized the NambuGorkov matrix representation [15] in (2) where the selfenergy matrix $\hat{\Sigma}$ is most generally written as an expansion $\hat{\Sigma}=\sum_{i} \Sigma_{i} \tau_{i}$ in terms of the Pauli matrices $\tau_{i}$. The diagonal components of $\hat{\Sigma}$ represent quasiparticle renormalizations, whereas the offdiagonal parts are nonzero in the superconducting state only.

Since the self-energy $\hat{\Sigma}(\mathbf{K}, \omega)$ does not depend on the integration variable $\tilde{\mathbf{k}}$, we can write

$$
\hat{\bar{G}}(\mathbf{K}, \omega)=\left(\omega \mathbb{1}-\bar{\epsilon}_{\mathbf{K}} \tau_{3}-\hat{\Sigma}(\mathbf{K}, \omega)-\hat{\Gamma}(\mathbf{K}, \omega)\right)^{-1},
$$

where $\bar{\epsilon}_{\mathbf{K}}=N_{c} / N \sum_{\tilde{\mathbf{k}}} \epsilon_{\mathbf{K}+\tilde{\mathbf{k}}}$. This has the form of the Green function of a cluster model with periodic boundary conditions coupled to a dynamic host described by $\hat{\Gamma}(\mathbf{K}, \omega)$. Here we employ the NCA to calculate the cluster Green function and self-energy respectively. A detailed discussion of the NCA-algorithm applied to the cluster model for the paramagnetic state was given in [12]. The NCA for the superconducting state has to be extended in order to account for the hybridization to the anomalous host, which couples cluster states with different particle numbers.

The self-consistent iteration is initialized by calculating the coarse grained average $\hat{\bar{G}}(\mathbf{K})$ (Eq. 目) and with Eq. 3 the host function $\hat{\Gamma}(\mathbf{K})$, which is used as input for the NCA. The NCA result for the cluster self-energy $\hat{\Sigma}(\mathbf{K})$ is then used to calculate a new estimate for the coarse grained average $\bar{G}(\mathbf{K})$ (Eq. 1). The procedure continues until the self-energy converges to the desired accuracy.

Results We investigate the single particle properties of the doped 2D Hubbard Model

$$
H=\sum_{i j, \sigma} t_{i j} c_{i \sigma}^{\dagger} c_{j \sigma}+U \sum_{i} n_{i \uparrow} n_{i \downarrow}
$$

where $c_{i}^{\dagger}\left(c_{i}\right)$ creates (destroys) an electron at site $i$ with spin $\sigma$ and $U$ is the on-site Coulomb repulsion. For the Fourier transform of the hopping integral $t_{i j}$ we use

$$
\epsilon_{\mathbf{k}}=\epsilon_{o}-\mu-2 t\left(\cos k_{x}+\cos k_{y}\right)-4 t^{\prime} \cos k_{x} \cos k_{y},
$$

accounting for both, nearest neighbor hopping $t$ and next nearest neighbor hopping $t^{\prime}$. We set $t=0.25 \mathrm{eV}$ and $U=3 \mathrm{eV}$, well above the bandwidth $W=8 t=2 \mathrm{eV}$. For this choice of parameters the system is a Mott-Hubbard insulator at half filling as required for a proper description of the high- $T_{c}$ cuprates.

To allow for symmetry breaking we start the iteration procedure with finite offdiagonal parts of the self-energy matrix $\hat{\Sigma}$. As we mentioned above one expects the order parameter of a possible superconducting phase to have $d$-wave symmetry. Therefore we work with a $2 \times 2$-cluster $\left(N_{c}=4\right)$, the smallest cluster size incorporating nearest neighbor correlations. For the set of cluster points we choose $\mathbf{K}_{\alpha l}=l \pi$, where $l=0,1$ and $\alpha=x$ or $y$. Fig.1 illustrates this choice of $\mathbf{K}$-points along with a sketch of the $d$-wave order parameter and the coarse graining cells. Obviously, for symmetry reasons, in the case of $d$-wave superconductivity, we expect the coarse grained anomalous Green function to vanish at the zone center 
and the point $(\pi, \pi)$. Whereas the anomalous parts at the points $(0, \pi)$ and $(\pi, 0)$ should be finite with opposite signs.
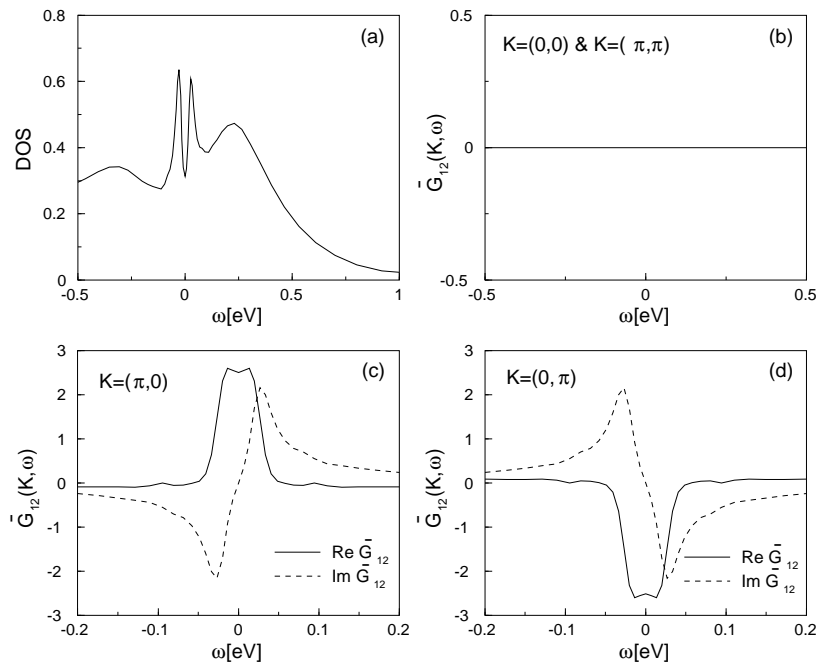

FIG. 2. (a) The local density of states (DOS) near the Fermi energy and the anomalous coarse grained Green functions at the cluster points (b) $\mathbf{K}=(0,0)$ and $\mathbf{K}=(\pi, \pi)$, (c) $\mathbf{K}=(\pi, 0)$ and (d) $\mathbf{K}=(0, \pi)$ in the superconducting state. The nearest neighbor hopping integral $t=0.25 \mathrm{eV}$, next nearest neighbor hopping integral $t^{\prime}=0$, bandwidth $W=2 \mathrm{eV}$, the on-site Coulomb repulsion $U=3 \mathrm{eV}$, temperature $T=137 \mathrm{~K}$ and the doping $\delta=0.19$. The anomalous parts of the Green function (b)-(d) are consistent with a $d$-wave order parameter.

Fig.2 shows a typical result for the local density of states (DOS) in the superconducting state along with the anomalous coarse grained Green function $\bar{G}_{12}(\mathbf{K}, \omega)=$ $N_{c} / N \sum_{\tilde{\mathbf{k}}}\left\langle\left\langle c_{\mathbf{K}+\tilde{\mathbf{k}} \uparrow} ; c_{-(\mathbf{K}+\tilde{\mathbf{k}}) \downarrow}\right\rangle\right\rangle_{\omega}$ at the cluster $\mathbf{K}$-points for $t^{\prime}=0$, temperature $T=137 \mathrm{~K}$ and doping $\delta=0.19$. The anomalous coarse grained Green function vanishes at the cluster points $(0,0)$ and $(\pi, \pi)$ but is finite at the points $(\pi, 0)$ and $(0, \pi)$, consistent with a $d$-wave order parameter. Note that this result is independent of the initialization of the self-energy, i.e. an additional initial $s$-wave contribution vanishes in the course of the iteration. Thus a possible $s$-wave contribution to the order parameter can be ruled out.

The finite pair amplitude is also reflected in the local density of states (DOS) depicted in Fig.22a, where we show the lower sub-band of the full spectrum near the Fermi energy. It displays a pseudogap at zero frequency as expected for a $d$-wave order parameter.

Fig. 3 shows the DOS near the Fermi energy for the same parameters as in Fig.2, fixed temperature $T=$ $137 \mathrm{~K}$, but for various dopings. Obviously, the pseudogap size, measured as the peak to peak distance, as well as the density of states at the Fermi energy do not depend strongly upon doping. However the drop in the density of states from the gap edge to the $\omega=0$ value first increases, reaches a maximum at about $19 \%$ doping, then decreases again.

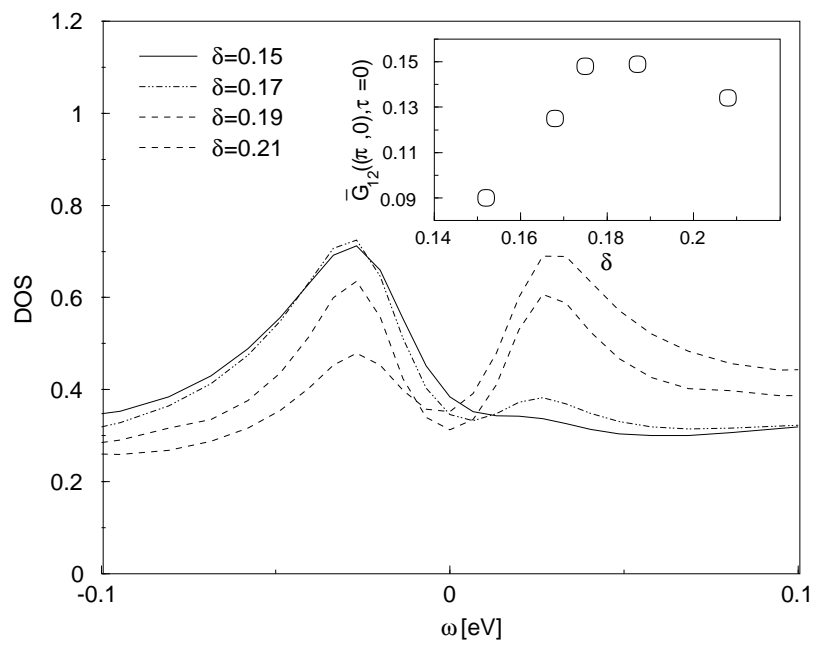

FIG. 3. Density of states in a narrow region at the Fermi energy for the same parameters as in Fig. 2 but for various dopings. The gap size and the density of states at $\omega=0$ are independent of doping. Inset: Equal time coarse grained anomalous Green function $\bar{G}_{12}(\mathbf{K}, \tau=0)$ at $\mathbf{K}=(\pi, 0)$.

This behavior originates in the doping dependence of the anomalous Green function. In the inset we plot the coarse grained anomalous equal time Green function $\bar{G}_{12}(\mathbf{K}, \tau=0)=N_{c} / N \sum_{\tilde{\mathbf{k}}}\left\langle c_{\mathbf{K}+\tilde{\mathbf{k}} \uparrow} c_{-(\mathbf{K}+\tilde{\mathbf{k}}) \downarrow}\right\rangle$ for $\mathbf{K}=(\pi, 0)$. This number as a measure of the superconducting gap shows exactly the same behavior as the pseudogap in the density of states.

The anomalous components $\bar{G}_{12}(\mathbf{K}, \omega)$ and hence the pseudogap in the DOS become smaller with increasing temperature and eventually vanish at a critical temperature $T_{c}$ depending on the set of parameters. The phase diagram is shown in Fig. 1 . As a function of doping, $T_{c}(\delta)$ has a maximum $T_{c}^{\max } \approx 150 \mathrm{~K}$ at $\delta \approx 19 \%$ and strongly decreases with decreasing or increasing $\delta$. The qualitative behavior of $T_{c}(\delta)$ in the calculated $T-\delta$ region agrees well with the generic phase diagram of the high- $T_{c}$ cuprates. Unfortunately, due to the break-down of the NCA at very low temperatures we are not able to extend the phase diagram beyond the region shown in Fig.4. This means in particular that we cannot predict reliable values for $\delta_{c}(T=0)$, beyond which superconductivity vanishes.

The inset of Fig. 1 shows the transition temperature dependence $T_{c}\left(t^{\prime}, \delta=\right.$ const. $)$ on the next nearest neighbor hopping amplitude $t^{\prime}$ for fixed doping $\delta=0.18$. As compared to $t^{\prime}=0 T_{c}$ strongly decreases with growing negative $t^{\prime}$ but increases for $t^{\prime}>0$. The shape of the phase diagram as well as the $t^{\prime}$-dependence of $T_{c}$ can be qualitatively understood in terms of the phenomenological picture, where spin fluctuations mediate the electronelectron interaction, which then is strong at the antifer- 
romagnetic wave vector $\mathbf{Q}=(\pi, \pi)$.

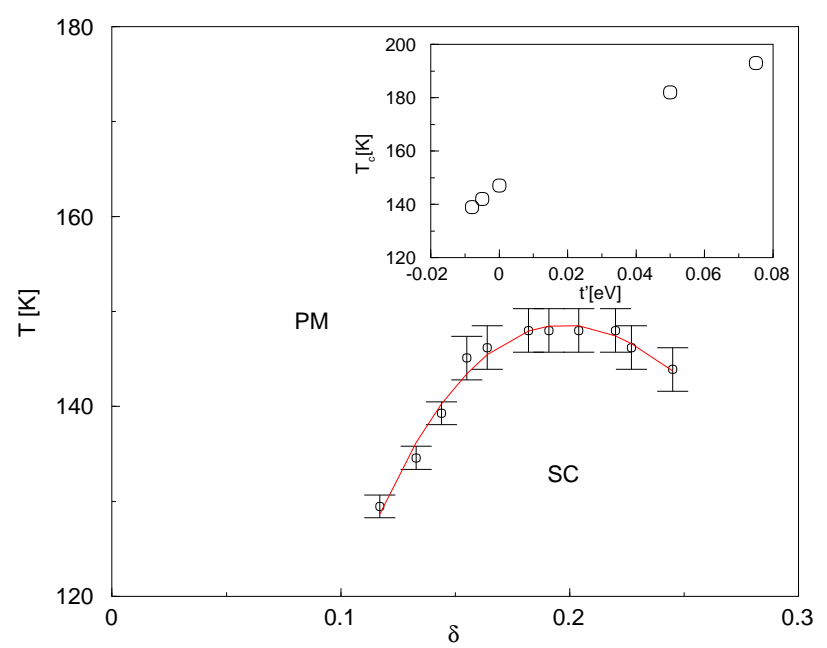

FIG. 4. Temperature-doping phase diagram for the $2 \mathrm{D}$ Hubbard model via DCA for a $N_{c}=4$ cluster. The nearest neighbor hopping $t=0.25 \mathrm{eV}$, next nearest neighbor hopping $t^{\prime}=0$ and the Coulomb repulsion $U=3 \mathrm{eV}$. The error bars result from the finite resolution in temperature. Inset: Transition temperature $T_{c}\left(t^{\prime}\right)$ for fixed doping $\delta=0.18$ as a function of the next nearest neighbor hopping amplitude $t^{\prime}$.

In Fig. 5 we display the coarse grained spectra $-\frac{1}{\pi} \Im m \bar{G}_{11}(\mathbf{K}, \omega)$ at $\mathbf{K}=(\pi, 0)$ in the normal state $(T=290 \mathrm{~K})$ for the next nearest neighbor hopping $t^{\prime}=0$ and $t^{\prime}=-0.05 \mathrm{eV}$ (left and right hand side) for different dopings $\delta$. At the bottom we show the Fermi surfaces of the corresponding noninteracting systems $(U=0)$ in the first quadrant of the BZ. The diagonal thick solid line indicates the set of $\mathbf{k}$-points which fulfill the nesting condition, i.e. which can be connected by $\mathbf{Q}$ to equivalent k-points in the opposite quadrant of the BZ.

The doping dependence of the $t^{\prime}=0$ and $t^{\prime}=-0.05$ spectra is qualitatively different. In the $t^{\prime}=-0.05$ case the parts of the Fermi surface near $\mathbf{K}=(\pi, 0)$ and $(0, \pi)$ fulfill the nesting condition roughly for the whole doping range, the quasiparticles couple strongly to the spin fluctuations and hence the corresponding spectra display a pseudogap at zero frequency over the entire doping range. The $t^{\prime}=0$ spectra in contrast exhibit the pseudogap in the underdoped regime only $(\delta=0.05)$, where the spin fluctuations are strong, but show a quasiparticle peak at optimal doping $\delta=0.19$, where the points on the Fermi surface near $\mathbf{K}=(\pi, 0)$ and $(0, \pi)$ are far from being nested. The suppression of the density of states at the Fermi energy results in a suppression of superconductivity and hence the transition temperatures drop with decreasing doping as well as decreasing $t^{\prime}<0$. For positive $t^{\prime}$ we obtain similar spectra and Fermi surfaces as for $t^{\prime}=0$, but with a slightly enhanced density of states at the Fermi energy, resulting in higher transition temperatures.

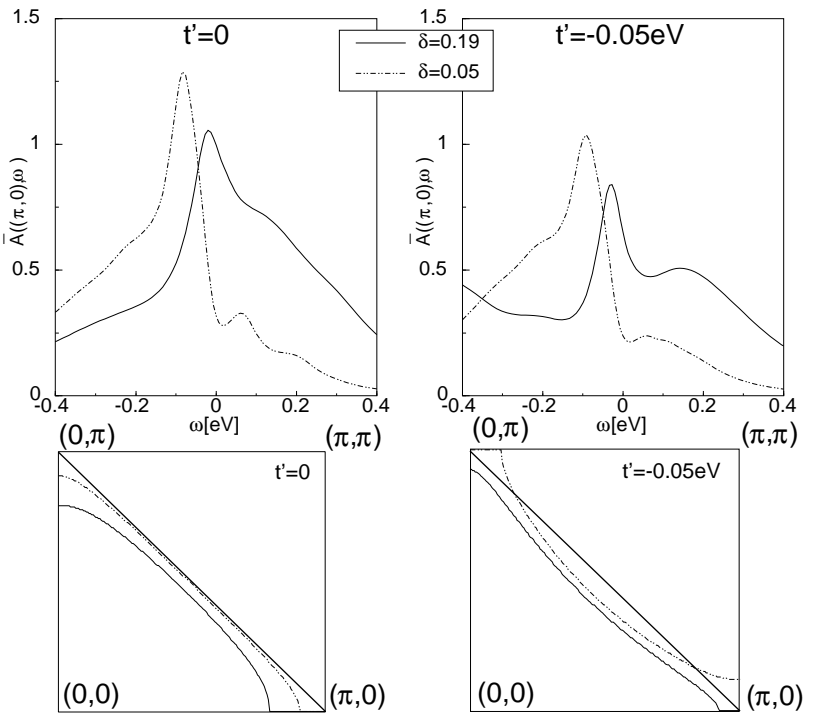

FIG. 5. Coarse grained spectral density $-\frac{1}{\pi} \Im m \bar{G}_{11}(\mathbf{K}, \omega)$ at $\mathbf{K}=(\pi, 0)$ in the normal state $(T=290 \mathrm{~K})$ for the next nearest neighbor hopping $t^{\prime}=0$ (left) and $t^{\prime}=-0.05 \mathrm{eV}$ (right) for different dopings $\delta$. The nearest neighbor hopping $t=0.25 \mathrm{eV}$ and the Coulomb repulsion $U=3 \mathrm{eV}$. Bottom: Fermi surfaces of the corresponding noninteracting $(U=0)$ systems in the first quadrant of the BZ. The thick diagonal line indicates the set of $\mathbf{k}$-points which fulfill the nesting condition.

Summary We have used the recently developed DCA to study the long open question of whether the 2D Hubbard model shows instabilities towards a superconducting state in the intermediate to strong coupling regime. We find conclusive evidence that at moderate doping a transition to a state with offdiagonal long range order occurs and that the corresponding order parameter has pure $d$ wave symmetry. The corresponding temperature-doping phase diagram agrees qualitatively with the generic high$T_{c}$ phase diagram.

Acknowledgements It is a pleasure to acknowledge useful discussions with P.G.J. van Dongen, M. Hettler and H.R. Krishnamurthy. This work was supported by NSF grants DMR-9704021, DMR-9357199 and the Graduiertenkolleg "Komplexität in Festkörpern". Computer support was provided by the Ohio Supercomputer Center and the Leibnitz-Rechenzentrum, Munich.

[1] For a review, see M.B. Maple, cond-mat/980202.

[2] H. Ding et al., Nature 382, 51 (1996).

[3] F. Ronning et al., Science (1998).

[4] For a review, see D.J. Scalapino, cond-mat/9908287.

[5] P. Monthoux, A.V. Balatsky and D. Pines, Phys. Rev. Lett., 67, 3448, (1991). 
[6] T. Timusk and B. Statt, cond-mat/9905219.

[7] P.W. Anderson, The Theory of Superconductivity in the High- $T_{c}$ Cuprates, Princeton University Press , Princeton, NJ (1997)

[8] N.E. Bickers, D.J. Scalapino, and S.R. White, Phys. Rev. Lett., 62, 961 (1989).

[9] T. Moriya, Y. Takahashi, and K. Ueda, J. Phys. Soc. Japan, 59, 2905, (1990).

[10] W. Metzner and D. Vollhardt, Phys. Rev. Lett. 62, 324 (1989).

[11] M.H. Hettler et al., Phys. Rev. B 58, 7475 (1998).

M.H. Hettler et al., preprint cond-mat/9903273.

[12] Th. Maier et al., Eur. Phys. J. B 13, 613 (2000).

[13] C. Huscroft et al., cond-mat/9910226.

[14] D.F. Elliot and K.R. Rao, Fast Transforms: Algorithms, Analyses, Applications (Academic Press, New York, 1982).

[15] J.R. Schrieffer, Theory of Superconductivity, Addison Wesley, Reading, MA (1993).

[16] H. Keiter, J.C. Kimball, Intern. J. Magnetism, 1, 233 (1971). 